\title{
Pediatric Multiple Sclerosis
}

\author{
Dorothée Chabas, Ari J. Green, and Emmanuelle Waubant
}

University of California at San Francisco, Multiple Sclerosis Center, San Francisco, California 94117

\begin{abstract}
Summary: Multiple sclerosis (MS) occurs at all ages of the pediatric population. Childhood MS may represent up to $10 \%$ of all MS cases. Establishing the diagnosis of MS in a child is complicated by the limited diagnostic criteria and the possibility of significant clinical and magnetic resonance imaging (MRI) overlap with acute disseminated encephalomyelitis and other pediatric diseases. Although the clinical profile of MS appears similar to that seen in adults, several features may differ and specific issues arise in children. Sex ratios are different between young children with MS and adolescentsimplicating a role for sex hormones in disease pathogenesis and/or modification of disease expression. Younger patients with MS are more likely to have seizures, brainstem, and cerebellar symptoms than adults. Children with MS may have
\end{abstract}

fewer T2 hyperintense areas on MRI scans, therefore not meeting MRI criteria established for adults. It is possible that the pediatric MS course is more indolent than in adult patients but the disease may lead to significant disability at a younger age, e.g., while patients are students, young professionals, or want to start a family. There has been no controlled clinical trial in children with disease modifying therapies approved for adult MS due to the limited number of patients under the age of 18 years compared with the adult contingent. As a result, children are receiving adult therapies in an arbitrary manner and our understanding of pediatric treatment effect and tolerability is limited. Available data on tolerability of approved drugs for adults is reviewed. Key Words: Multiple sclerosis, acute disseminated encephalomyelitis, pediatric, treatments, review.

\section{INTRODUCTION}

Multiple sclerosis (MS) is a prototypical demyelinating disease of the CNS in adults, affecting approximately 400,000 individuals in the United States. This autoimmune disease has a complex etiology likely related to both genetic and environmental factors. Its protean manifestations include visual, motor, sensory, and cognitive impairment but MS can in fact impact nearly any neurological function. ${ }^{1}$ As many as $80 \%$ of adult MS patients experience initial episodes of acute worsening followed by clinical remission, hence the name "relapsingremitting" (RR) MS. ${ }^{2}$ A sizable portion of these patients convert to a more progressive course later in their disease. During this secondary progressive (SP) MS course, disability accumulates significantly over time regardless of clinical exacerbations. Approximately $10-15 \%$ of adult MS patients have insidious progression from the onset of disease ("primary progressive" (PP) MS). A much smaller subset initially has a progressive course and later develops rare clinical exacerbations ("progres-

Address correspondence and reprint requests to Dorothée Chabas, M.D., Ph.D., University of California at San Francisco, Multiple Sclerosis Center, 350 Parnassus Avenue, Suite 908, San Francisco, CA 94117. E-mail: dorothee.chabas@ucsf.edu. sive-relapsing" (PR) MS). MS primarily affects young adults with a peak incidence between the ages of 20 and 40 and is the leading cause of nontraumatic neurological disability in this population in the industrialized world. However, MS can also be diagnosed in patients from ages 2 to 80 . In fact, because clinicians fail to recognize the disease in patients who do not fit the typical age profile, MS is probably underdiagnosed in the pediatric population.

Saint Ludwina of Schiedem is often cited as the first historically recorded case of MS. As a teenager in Holland during the 14th century she developed sensory symptoms and visual loss with a relapsing remitting course-ultimately becoming physically disabled and blind. ${ }^{3}$ Still 500 years later the original medical descriptions of MS by Charcot highlighted the disease as a condition afflicting young adults. This propensity for the disease to occur in people between ages 20 and 40 led Kurtzke to declare that "any disease beginning before age 10 should be called MS only as a last resort." As a result, successive diagnostic criteria for MS imposed age requirements that we have since realized were excessively restrictive. In fact, recent descriptions of the full clinical spectrum of MS suggest that as many as $1 \%$ of cases present in the first decade of life and up to $15 \%$ 
have clinical onset before age $18{ }^{5,6}$ Although, we now recognize that MS occurs in the pediatric population, there remain many unanswered questions about the disease in young patients. This review will attempt to describe those differences, warn clinicians as to pitfalls in the diagnosis of MS in a pediatric population, and describe what we know about the use of disease-modifying treatments (DMT) in this population.

\section{DIAGNOSIS OF MS}

Multiple sclerosis onset is defined by the occurrence of the first neurological symptoms of CNS dysfunction. In children, this first episode can be either dismissed when mild or misleading, e.g., diagnosed as acute demyelinating encephalomyelitis (ADEM) or another disorder. The diagnosis of MS requires dissemination in time and in space. ${ }^{7}$ For this reason, it is often established only after the second clinical attack. However, in many cases, typical findings on serial magnetic resonance imaging (MRI) scans facilitate early diagnosis, at least in adults, as changes can account for dissemination of the disease in time. ${ }^{8}$ MRI findings include T2 bright ovoid areas in the deep white matter that sometimes enhance on T1weighted sequences after administration of gadolinium as a result of transient blood-brain barrier breakdown at the time new lesions appear. Interestingly, disease activity identified by neuroimaging does not always correlate with clinical symptoms and disability. Only lesions located in physiologically relevant areas and of sufficient size give rise to neurological symptoms. In fact, for every new clinical attack, 5 to 10 new plaques may be detected on serial brain MRI scans. ${ }^{9}$ The presence of intrathecal inflammation, e.g., presence of specific oligoclonal bands or elevated IgG index in the spinal fluid compared to serum, also helps to establish the diagnosis. It is unclear whether all of the components of the diagnostic criteria used in adult patients are also applicable to children. ${ }^{10}$ The clinical and MRI overlap with other diseases, particularly ADEM, remains a vexing problem with important prognostic implications in the cases of individual patients, while raising challenging scientific questions pertaining to the monophasic versus remitting course of both disease entities.

\section{PEDIATRIC MS FEATURES}

Many attempts have been made over the last 30 years to characterize the features of MS in childhood-most of them by describing the experience of individual centers around the world. Despite the limitations of these studies they provide important insight into pediatric MS and its mimics. As the volume of these studies has grown, suggestions of significant differences between MS in the adult population and in children have become apparent.
Advances in imaging over the last 10-20 years have increased the recognition of MS in the pediatric population and led to more reliable diagnoses. This in turn has resulted in larger more reliable series in the same period. However, it seems questionable that identical MRI criteria as in adults can be used in pediatric MS. ${ }^{10}$

Conceptually, there are differences between the pediatric and adult populations that could explain at least in part clinical and MRI specificities in younger patients. Examples include more recent exposure to common environmental factors such as viruses, immature immune system, better capacity for CNS repair, and different ability to tolerate medications.

\section{Clinical aspects}

Practical and sociocultural factors complicate the recognition of MS in children. Subjective and transitory symptoms in children may be overlooked or incompletely investigated. In addition, measuring visual loss or somatosensory deficits in young children may be difficult. Concentration problems or fatigue may be attributed to attention deficit disorder or nonorganic causes. These factors contribute to a potential underreporting bias of mild cases in the pediatric MS literature and also may delay the identification of MS until adolescence or adulthood.

In addition, similar to the diagnostic issues faced in the adult population, significant clinical overlap with other pediatric neurological disorders and MS can make the diagnosis difficult (see Table 1). Other diseases can also demonstrate a similar capacity to "relapse" and "remit" (e.g., recovery after pediatric stroke). In fact, it is widely recognized that some of the patients described by Schilder most likely did not have MS. ${ }^{21}$ It has been suggested that misdiagnoses and referral bias have led to the false impression that pediatric MS is more virulent than its adult counterpart. ${ }^{11}$

Many observers have attempted to delineate clinical differences in the presentation and expression of MS between children and adults. Most of these studies are retrospective case series that provide no opportunity to capture subtle or complicated diagnoses that were missed. ${ }^{5,6,12-20}$ Many of them have used different diagnostic criteria and have designated cases as "pediatric" based on different age cut-offs (ranging from 10 to 20 years). For example, Sindern and colleagues ${ }^{5}$ reported on the 31/620 MS patients admitted to a neurology service who had onset before age 16. They used Poser's criteria for diagnosis and considered CSF positive with four oligoclonal bands. ${ }^{7}$ In contrast, Duquette et al. ${ }^{12}$ reported on patients with onset before age 16 but used Rose et al.' $\mathrm{s}^{22}$ criteria for diagnosis, and patients, more typically, did not require hospital admission for inclusion.

Some early reports noted differences that were likely a product of bias caused by the small number of cases in 
TABLE 1. Differential Diagnosis for Pediatric MS

\begin{tabular}{|c|c|}
\hline Clinical Presentation & Differential Diagnosis \\
\hline $\begin{array}{l}\text { Progressive neurological decline }+ \text { tumefactive lesion } \\
\text { on MRI }\end{array}$ & Lymphoma, medulloblastoma, other malignancy or infection \\
\hline $\begin{array}{l}\text { Progressive neurological decline plus: } \\
\text {-developmental delay, } \\
\text {-family history of white matter disorder, } \\
\text {-involvement of peripheral nervous system and extra } \\
\text { neural organ, } \\
\text {-widespread confluent white matter lesion on MRI }\end{array}$ & $\begin{array}{l}\text { Metachromatic leukodystrophy, Fabry disorder, childhood } \\
\text { ataxia with cerebral hypomyelination }\end{array}$ \\
\hline $\begin{array}{l}\text { Intermittent or progressive neurological decline, plus } \\
\text { diabetes or other metabolic abnormalities, hearing } \\
\text { loss, myopathy, cardiomyopathy, vision loss }\end{array}$ & Mitochondrial disorders, Alexander's disease \\
\hline $\begin{array}{l}\text { Developmental delay, transient neurological dysfunction, } \\
\text { including seizures, ataxia, extra neural involvement }\end{array}$ & Aminoaciduria \\
\hline Spinal cord dysfunction & $\begin{array}{l}\text { Acute transverse myelitis, neuromyelitis optica (Devic's } \\
\text { disease), spinal cord tumor }\end{array}$ \\
\hline Nonspecific white matter changes on MRI & Nutritional disorders \\
\hline $\begin{array}{l}\text { Transient or long lasting neurological deficit suggestive } \\
\text { of TIA or stroke with headache or migraine }\end{array}$ & $\begin{array}{l}\text { Antiphospholipid antibody, migraine, AVM, Moya Moya } \\
\text { syndrome }\end{array}$ \\
\hline Encephalopathy & Herpes virus infections or others, Lyme disease \\
\hline Myelopathy & HLTV- 1 or -2 \\
\hline Encephalopathy and movement disorder & Post streptococcal infection \\
\hline $\begin{array}{l}\text { History of rheumatological disease, joint pain and } \\
\text { swelling, skin lesions, kidney disease }\end{array}$ & $\begin{array}{l}\text { Systemic lupus erythematosus, antiphospholipid antibody } \\
\text { syndrome, vasculitis, sarcoidosis, Behcet's disease }\end{array}$ \\
\hline
\end{tabular}

$\mathrm{AVM}=$ arteriovenous malformations; HLTV $=$ human $\mathrm{T}$-cell lymphotropic virus.

the series. ${ }^{14,15}$ In larger, more recent studies, potential biases (beyond the issues of retrospective series) include difficulties associated with recognizing certain clinical syndromes in children (e.g., sensory or mild cognitive complaints as mentioned above) and population differences between the reporting clinic and the general MS population (especially in those studies that did not make comparisons to their own local adult MS population.) Given that ethnic differences in MS expression have been increasingly recognized (i.e., the differences between MS in Caucasian, African-American, and Asian populations) and the almost universal acknowledgment of genetic factors in disease expression-one could imagine that comparisons between certain pediatric populations and the universal adult MS population may reflect genetic factors as much as they reflect age-related differences. Still, a number of important clinical differences have been repeatedly described in papers describing "pediatric MS" and appear to be biologically plausible. The distribution of some clinical findings has been reported to be different in children. The most believable observations are that young children with MS are more likely to develop seizures and/or ataxia., ${ }^{5,12,17,18}$

According to several observers mean time to recovery after a clinical exacerbation appears to be shorter in children compared with adults (4.3 weeks versus $6-8$ weeks in adults). ${ }^{20,23}$ In addition, comparing children under 15 to three groups of older adults from the same center, Trojano et al. ${ }^{23}$ noted that the children in their study had a higher relapse rate, shorter interval between their first and second attack, and yet a lower overall disability level than adult patients with the same disease duration. The rapid recovery and reduced overall disability seen in children argue that some factors about a child's nervous system make them more conducive to recovery. One might attribute this to the resilience of the childhood CNS. However, elucidating the mechanisms by which the young nervous system recovers from an attack of demyelination (whether it be by extensive and rapid remyelination, redistribution of sodium channels, or reorganization of functional pathways) and understanding whether the child's brain may have significant differences in the inflammatory milieu or reduced axonal injury would provide important insights into the nature of clinical recovery in MS.

The shorter duration between attacks observed by Trojano and coworkers ${ }^{23}$ argues that possibly some MS cases present in childhood because of a more aggressive autoimmune process (as opposed to random chance or earlier exposure to an environmental factor). This contention is further supported by the increased presence of large or tumefactive lesions in pediatric cases (further discussed below).

The proportion of patients with progressive disease from onset may also be different in childhood MS. In adults, PP and PR MS represent up to $20 \%$ of patients, whereas in childhood MS disease progressive from onset is rare (e.g., less than 7\%).,17,24 Although it is not clear what these differences reflect, there is a notion that patients with disease progressive from onset are typically 
older, and the male:female ratio approaches 1. Further, some subtypes of MS defined by biological findings ${ }^{25}$ may be more frequent in specific age categories, suggesting that neurodegenerative mechanisms could be more frequent in older patients.

There appears to be two relatively distinct age-related subsets of pediatric MS patients. These might best be described as childhood MS (age 2-10 years) and adolescent-onset MS (age 10-18 years). The exact age for distinction is somewhat arbitrary and reflects the artificial and consensual World Health Organization definition of adolescence. Although there seems to be clinical differences betweens these two populations, the most striking one being the relative increase in male cases in patients younger than age 10 (closer to parity in sex distribution as opposed to the 1.5-2:1 female preponderance in adult patients). ${ }^{5,12,24}$ The difference observed may reflect the importance of pubescence and sex hormones in MS pathogenesis in the older population. Further support for this interpretation of the data is provided by the remarkable increase in female/male ratio in patients around pubescence (2-4:1 in patients $12-15$ years at onset). Some studies have reported the increase seen in the pubescent population as true for all pediatric MS cases, but largely because they have collapsed all patients younger than 16 or 15 years into a single group. Re-evaluation of the sex distribution in their small groups of younger than 10-year-old patients supports the relative parity mentioned above. ${ }^{5,12,17,18,26}$

Overall, regarding physical disability, the prognosis for pediatric MS cases appears relatively good. They appear to have less physical disability compared with adult patients who have suffered the same disease duration, but appear to be worse off compared with adult onset patients at the same age (presumably because of longer disease duration). In Duquette and coworkers'series more than three-fourths of the patients were still walking at 15 years. Ghezzi's series reported that around $25 \%$ of cases had EDSS $>6.0$ after longer than 8 years follow-up. ${ }^{5,12,24}$ In addition to the sex distribution differences, it is possible that childhood MS might be less physcially disabling than either adult or adolescent MS, with few patients exhibiting permanent disability after similar disease duration; although the difference between children and adolescents has never been statistically demonstrated but is hypothetized by the authors based on their personal experience.

Most physical symptoms are transitory and remit more quickly in children than in adults. Paradoxically, extremely early onset of MS ( $<2$ years of age) appears to be associated with a very poor prognosis, although the small number of observed cases makes extrapolation unreliable. ${ }^{4,18,27}$ Finally, one has to keep in mind that, as opposed to physcial disability, cognitive disability may be more profound for pediatric MS patients than for adult-onset MS patients and involve school disruption and other cognitive issues specifically related to that age range and can be particularly disabling.

Indeed, one manner in which pediatric MS has recently been recognized to be more similar to adult disease is in the spectrum of cognitive impairment in children with the disease. This obviously has serious implications in individuals undergoing development of their cognitive activities. ${ }^{28,29}$ Banwell and Anderson ${ }^{28}$ reported recently on a small group of adolescent MS patients with little motor disability, all of whom had difficulty on one of a number of neuropsychiatric tests administered. Although some of the observations may be confounded by fatigue and/or depression and extrapolation from this small group may be difficult, it establishes that further investigation into the cognitive burden of MS in children is warranted. ${ }^{28}$ In contrast, children with a remote history of ADEM ( $>2$ years prior) have been found to have less-pronounced cognitive impairments and typically perform within the normal range for their age group. Only visuospatial impairments appeared to be relatively common in a small study that evaluated 15 ADEM patients. $^{30}$

Finally, some clinical series provide evidence of ethnic variability in pediatric MS incidence and course. A large series of 256 patients from China found that $3.5 \%$ of Chinese MS patients developed symptoms before the age of 10 and $22 \%$ developed symptoms before the age of $20 .{ }^{31}$ Other smaller series from Asia provide support for the idea that Asian MS patients are more likely to have onset before age $20 .{ }^{32-35}$ There has also been the intriguing observation that - similar to what has been observed in adult patients-MS in African-American and West Indian children may follow a more debilitating course than in Caucasians. ${ }^{36,37}$

\section{MRI aspects}

The degree to which pediatric MS patients share imaging characteristics with adult patients is also controversial. The applicability of imaging criteria in the pediatric population and the utility of using these criteria for establishing a diagnosis is uncertain. Hahn and colleagues ${ }^{10}$ report that only 53 and $67 \%$, respectively, of their pediatric patients who ultimately developed clinically definite MS fit McDonald criteria for dissemination in space at the time of their initial and second attacks. ${ }^{8}$ This may be because the McDonald criteria, especially in the absence of gadolinium enhancement, have a requirement for at least three to nine total lesions depending on their location and enhancement status. It may be that the shorter period over which lesions can silently accrue in pediatric MS translates into a smaller number of total lesions at presentation. A relatively high incidence of tumefactive lesions seems to occur in pediatric MS compared to adults. ${ }^{10}$ 
It has also been claimed that imaging during workup for pediatric MS is useful for distinguishing ADEM from the first attack of MS. ${ }^{19}$ In young patients with an initial demyelinating event, the presence of long axis callosal lesions as well as well-defined focal lesions appear helpful in distinguishing CDMS from monophasic disease. ${ }^{38}$ However, none of these findings are specific for pediatric MS on their own and none are seen with uniform frequency. Combining the former two criteria (well-demarcated focal as well as long axis callosal lesions) achieves a very high specificity but poor sensitivity to diagnose MS. ${ }^{38}$ Other differences between ADEM and MS lesions in terms of lesion distribution have been described, such as ADEM lesions are more frequently seen in cortical gray mater and are much more commonly seen in subcortical white matter (WM) rather than periventricular WM. ${ }^{19,39,40}$ It is likely that the distinction between MS and ADEM can only be made with clinical follow-up and repeat imaging. The McDonald criteria, validated for diagnosing MS in the adult population and including MRI features, could be appropriate for defining MS in the population of children experiencing a first demyelinating event. However they have not been validated yet in that particular population.

\section{Biological aspects}

The CSF profile for pediatric MS cases appears to be similar to that found in adults, with the exception of a slightly higher incidence of pleocytosis in children. Pohl and coworkers ${ }^{41}$ have reported a very high sensitivity ( $>$ $90 \%$ ) for detecting oligoclonal bands in known pediatric MS cases between ages 6 and 16 years. Other studies have suggested that a much lower rate of oligoclonal bands is found in very young patients $(<10 \%)$. It is unclear whether this is the product of a shorter disease course rather than a difference of some basic factor influencing antibody production in very-early-onset MS. ${ }^{18}$

One possibility is that MS in children is identical to its adult counterpart except that the clinically symptomatic lesion occurs earlier in pediatric cases (leading to recognition and diagnosis). This contention is supported by the observation that in many cases a smaller overall burden of disease is seen on imaging in pediatric cases compared with adults. ${ }^{10,38}$ However, some clinical and imaging features argue that there are more profound differences between MS in children and adults. For example, it has been reported that a substantial minority of MS cases in children present with encephalopathy-which is almost never seen in adults. ${ }^{5,12}$ The clinical significance of this observation remains unclear. It may reflect a basic difference between adult and pediatric MS or, alternatively, it may reflect the diagnostic difficulty of differentiating MS from ADEM and the much increased relative frequency of the latter entity in a pediatric population. It may also reflect an increased sensitivity of the child's brain to insults in certain areas of the CNS.

Information gleaned from studying childhood MS has important implications for MS epidemiology in general. The increased burden of disease in females right around the time of menarche and the declining sex difference ratio in middle age argues for a role for sex hormones in causing or modifying the disease. The role of environmental agents such as viruses may be best studied in childhood MS, especially if these viruses are common and most individuals have been infected by adulthood. In support of the role of viral exposure in children, recent work reports that pediatric MS patients are much more likely than matched controls to be positive for EpsteinBarr virus. ${ }^{42}$ If confirmed, these types of data may significantly advance our understanding of the disease.

\section{PEDIATRIC MS TREATMENTS}

Although medications that modify the course of MS have not been formally evaluated in patients under the age of 18 years, pediatricians and pediatric neurologists have initiated those treatments in attempt to control the disease in young patients. Treatments for MS include drugs that promote recovery from ongoing exacerbations and drugs that reduce the frequency of relapses and, therefore, have the potential to slow down the progression of disability.

\section{Treatment of relapses}

Relapse therapy shortens relapse length thereby limiting consequences on daily activities. ${ }^{43-45}$ Relapse treatment does not influence the risk of subsequent relapse or progression of disability. Thus, disabling relapses are often treated, whereas mild or spontaneously recovering relapses usually are not. No trial has been conducted for treatment of relapses occurring in children with MS. Therefore, relapses in children are treated like adult relapses with pulse glucocorticosteroids, or less often with plasma exchanges or intravenous immunoglobulins (IVIG).

Relapse therapy in adult MS consists in high doses of glucocorticosteroids (500 $\mathrm{mg}$ to $1 \mathrm{~g}$ i.v. methylprednisolone (IVMP) daily for 3-5 days). An oral taper over a 10- to 15-day period may be used for severe relapses. Lower doses of glucocorticosteroids appear to be less efficient than higher doses. ${ }^{46,47}$ Equivalent high doses of oral glucocorticosteroids may work as well as high doses of intravenous injections. ${ }^{48}$

In pediatric MS patients, glucocorticosteroid regimen is adapted as a function of weight and usually varies from 20 to $30 \mathrm{mg} / \mathrm{kg} /$ day of IVMP for 3-5 days, depending on relapse severity, tolerability, and cumulated dose of corticosteroid. Infusions do not necessarily require hospitalization, although blood pressure, urine glucose, and se- 
rum potassium need to be monitored. Rare side effects of high doses of IVMP, which include irritability, appetite changes, and insomnia, are reported. ${ }^{47}$ Gastric toxicity can be systematically prevented with gastric protection. High cumulated doses of glucocorticosteroids expose a higher risk of side effects, which might be of particular importance at this age. Corticodependence (adrenal deficiency) and, less frequently, corticoresistance (recurrence of neurological symptoms after corticosteroid tapering) may occur. Other steroid side effects such as growth retardation are more specific to the pediatric population. These long-term side effects can be prevented by avoiding long-term corticotherapy, repeated pulses, and prolonged progressive tapering when not necessary.

Plasma exchange is used for severe relapses in adult MS and Devic's disease (also called neuromyelitis optica) recovering poorly after pulses of high doses of glucocorticosteroids. ${ }^{49}$ Plasma exchange has not been validated in the pediatric MS population but has been reported as a potential alternative for severe relapses resistant to corticosteroids. ${ }^{50}$

Finally, IVIG has also been used for pediatric MS relapses poorly responsive to high doses of IVMP or in children who have a contraindication to glucocorticosteroids; whereas no clear evidence of efficacy has been reported for adults. ${ }^{51-57}$

\section{Disease-modifying therapies}

Approved DMTs for MS decrease the risk of subsequent relapses and disability progression in patients with relapsing MS. The impact of currently approved DMTs on disability progression seems mostly related to prevention of exacerbations with their potential for accumulation of residual disability. Approved immunomodulating treatments including interferon- $\beta$ (IFNB) and glatiramer acetate (GA) decrease the risk of clinical relapse by approximately $30 \%$, and the number of new lesions on brain MRI scans by $30-80 \%$ in adult patients. ${ }^{58-62}$ There is accumulating evidence that early treatment with DMT significantly influences disease course and limits accumulation of disability. ${ }^{63-65}$ The immunosuppressive drug mitoxantrone has also been approved for "worsening MS" (e.g., severe RR or SP MS leading to rapid disability besides immunomodulating treatments). ${ }^{66}$ Other drugs are used off-label to treat MS, in adults such as monthly pulses of high doses IVMP, oral azathioprine, intravenous cyclophosphamide, oral methotrexate, oral mycophenolate mofetil, IVIG, and plasma exchange. Natalizumab (Tysabri) was granted FDA approval in 2004 but was removed from the market in February 2005 for further investigation due to two cases of progressive multifocal leukoencencephalopathy. ${ }^{67-69}$ This drug is administered as a monthly intravenous infusion and has been shown to decrease the relapse rate by $60 \%$ and disability progression by $40 \%$ over a 2 -year period. ${ }^{70-75}$ It is unknown whether and when it will become available again.

No medication at this time has shown a significant impact on the progressive course of MS (e.g., regardless of exacerbations). ${ }^{76-83}$ As progressive disability progression (e.g., independent of exacerbations) rarely begins during childhood, it is crucial in these patients to prevent relapses during the relapsing-remitting phase of the disease and hope that treatments also delay entry into the SP phase.

Patients who participated in pivotal clinical trials of DMT for MS were all older than 18 years. At this time, because of the small number of pediatric MS patients and the proven efficacy in adult patients with MS, it is unlikely that there will be clinical trials in children to test the efficacy of approved drugs for adults. In fact, the efficacy of early DMT use on long-term disability ${ }^{63-65}$ has prompted physicians to treat pediatric MS patients with drugs approved in adult MS. Several recent pediatric cohorts suggest that DMTs are well tolerated and that the side effects are overall similar to those observed in the adult population (Table 2).

Immunomodulators. Interferon- $\boldsymbol{\beta}$. After intramuscular or subcutaneous injections, IFNB binds to specific receptors located on the surface of immune cells and induces a Th2 bias of the immune response, attenuates Th1 inflammation, reduces the penetration of immune cells through the blood-brain barrier, and thus prevents CNS inflammatory lesions. ${ }^{95}$

Two IFNB-1a (Avonex, Biogen Idec, Cambridge, MA; Rebif, Serono, Geneva, Switzerland) and one IFNB-1b (Betaseron, Berlex, CA) have been approved by the FDA for adult relapsing-remitting MS. Intramuscular IFNB-1a (Avonex) was also recently approved for adults presenting a first demyelinating event. Treatment with IFNB decreases relapse rate by $30 \%$ and MRI activity by $60-80 \%$ compared with placebo. ${ }^{58,60,61}$ Two studies of dose comparison suggest that higher-dose, higher-frequency IFNB therapy is associated with higher therapeutic effect over the short term (e.g., 1 or 2 years). ${ }^{96,97}$ However, higher dose higher frequency IFNB treatment is also associated with development of neutralizing antibodies against IFNB in at least $25 \%$ of patients within 1 or 2 years of treatment. ${ }^{98}$ It is now clear that the development of neutralizing antibodies is associated with a significant decrease of therapeutic effect. ${ }^{98}$

Although several studies in children and adolescents with MS suggest that interferon is overall well tolerated (Table 2), four cases of serious adverse events have been reported $^{90,91}$ : one case of systemic reaction, one case of polyarthritis, and two cases of depressive mood disorder. Other side effects in pediatric MS patients include flulike syndrome, headaches, and injection-site reactions (e.g., erythema and local edema) and are reported roughly within the same proportion as in the adult pop- 
TABLE 2. Cohort Studies and Case Reports of Tolerability of Interferon- $\beta$ and Glatiramer Acetate in Pediatric MS Patients

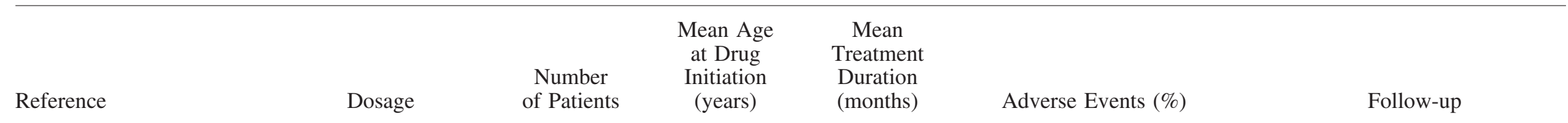

Interferon $\beta 1 \mathrm{~b}, \mathrm{~s}_{84} \mathrm{c}$.

Tenembaum et al.

Adams et al. ${ }^{85}$

Banwell 2005

Interferon $\beta$ 1a, i.m.

Waubant et al. ${ }^{87}$

Mikaeloff et al. ${ }^{88}$

Ghezzi et al. ${ }^{89}$ (ITEMS

study)

Interferon $\beta$ 1a, s.c.

Pohl et al 90
Schilling et al. ${ }^{86}$

Segura ${ }^{91}$

Etheridge et al. ${ }^{92}$

Glatiramer acetate, s.c.

Kornek et al. ${ }^{93}$
8 million units eod

43

4 million units eod

8 million units eod

1 (SPMS)

9

$30 \mu \mathrm{g} /$ week

progressively*

$30 \mu \mathrm{g} /$ week* $^{*}$

13

22

22-44 $\mu \mathrm{g} 3$ times

a week

51

24

$\mu \mathrm{g} 3$ times

week*
$7.3 \mu \mathrm{g} 3$ times a

week
13

7
15

$$
15
$$

$10.5-17$

$<16$

14.6

(n)

$20 \mathrm{mg} /$ day

7

43

16.6

$<18$
FLS 35, ALF 26, ISR 21

None

FLS

FLS 44, headache 44, fever 22, ISR 11

FLS around 69 ISR around 19

2
FLS 65, ALF 35, ISR 71, gastro-intestinal symptoms 10, blood count abnormalities 39 . 2 serious adverse effects

2 serious side effects ${ }^{\S}$

$7 \quad 24$

24
25 discontinued (1 for AE, 4 for lack of adherence, 5 for lack

of efficacy)

No further relapse

Neurological improvement

None discontinued because of $\mathrm{AE}$

$31 \%$ discontinued for lack of efficacy

Trend to decrease ARR

9 discontinued ( 6 for AE, 1 for lack of efficacy); trend to decrease ARR

1 discontinued for $\mathrm{AE}$, trend to decrease ARR

4 relapses on treatment

3 discontinued for lack of efficacy (none for AE)

1 discontinued because of AE

All patients reported in these studies had RR MS, except the patient reported by Schilling et al. ${ }^{112}$ who had SP MS.

FLS = Flu-like symptoms; ALF = alteration of liver functions; ISR = Injection site reaction; ARR = annualized relapse rate

${ }^{\dagger}$ In each of these studies, one patient used lower doses of interferon because of young age $(*)$ or because of an alteration of liver functions ( $\left.\dagger\right)$. All of these patients were among the youngest $(8,10$, and 12 years old, respectively).

Two cases of serious adverse effects were reported: one 12-year-old boy experienced a systemic reaction (edema, weakness, and mild pleural effusion), after 4 weeks on treatment ( $22 \mu \mathrm{g} 3$ times per week), then the symptoms disappeared within 2 weeks after discontinuation of treatment. Another 12- year-old boy developed a depressive mood disorder after 5 months on treatment ( $44 \mu \mathrm{g} 3$ times per week).

${ }^{\S}$ Two cases of serious adverse effects were reported: one patient developed a depressive mood disorder (and attempted suicide) after 1 year on treatment. Another patient developed polyarthritis after 3 months on treatment. 
ulation (see Table 2). Alteration of liver function [increased aspartate aminotransferase (AST) and alanine aminotransferase (ALT)] may be more frequent in young patients (younger than 10 years). ${ }^{84}$ However, potential side effects that might specifically pertain to the pediatric population (e.g., effect on growth, puberty, and the immature immune system) have yet to be evaluated. Also, studies in children have been mostly retrospective, and there is an inherent bias to underevaluate side effects in this setting. Finally, in adults, very long-term adverse events of IFNB remain unknown, as it became available only 12 years ago.

The data reported from various cohorts of children exposed to IFNB suggest that IFNB may decrease relapse rate in children with MS; however these studies were not designed to evaluate treatment efficacy and cannot tell us much about the IFNB therapeutic effect in patients younger than 18 years ${ }^{84,87-91}$

Glatiramer acetate. Glatiramer acetate (Copaxone, Teva-Marion Partners, Kansas City, MO) is an immunomodulating treatment approved for adult relapsing MS. It is made of a random polypeptide (L-glutamic acid, Llysine, L-alanine, and L-tyrosine), which induces an antigenic cross-reactivity with myelin protein reactive $\mathrm{T}$ cells. GA induces the production of anti-GA-regulating Th2 T cells that regulate CNS inflammation (by standard suppression). ${ }^{95}$ It is unclear whether GA has neuroprotective properties. GA is administered daily as $20 \mathrm{mg}$ s.c. injections. GA decreases the rate of relapses and new MRI lesions by approximately $30 \% .{ }^{59,62}$ Its effect on progression of disability is unclear.

As for IFNB, preliminary safety data suggest that GA is well tolerated in the pediatric MS population (Table 2). ${ }^{93,94}$ There are fewer side effects with GA than with IFNB. There are however no data available on potential long-term adverse effects, and GA efficacy has not been evaluated in the pediatric population.

\section{DMT prescription guidelines}

There is no consensus for the use of DMT in pediatric MS. For several reasons, an increasing number of pediatric MS patients are being offered DMTs approved for adults, despite the lack of validation. First, pediatric MS seems to be the same disease as adult MS. Second, DMTs approved for adults seem to have similar side effect profiles in children and adolescents. Finally, there is growing evidence that early treatment may influence disease course over the long term. ${ }^{63-65}$

As in adults, IFNB should be titrated over a period of 1 month to maximize tolerability, particularly for Rebif and Betaseron. It is common to use one-quarter dose for the first 2 weeks, one-half dose for the next 2 weeks, and the full dose subsequently if tolerated. Most of the time doses similar to those used in adults are well tolerated, particularly in adolescents. ${ }^{84,91}$
Dosage may be reduced according to tolerability and low weight of the patient. It is recommended to monitor complete blood count and liver function (AST, ALT) at baseline, monthly for the first 3 months, and every 3 months subsequently as IFNB has potential liver toxicity. GA is used readily at the full dosage (20 $\mathrm{mg} /$ day) as in adults. ${ }^{93,94}$ In contrast to IFNB, GA does not require laboratory monitoring. For all DMT, it is critical to evaluate compliance. In young children, treatment is generally administered by parents, whereas, in teenagers, it is often self-administered. Involving the child as much as possible in treatment decision and initiation promotes compliance. ${ }^{99}$

An international task force sponsored by the National Multiple Sclerosis Society is currently working on consensual definitions and therapeutic guidelines for pediatric MS.

\section{Intravenous immunoglobulins}

Several controlled trials in adults have reported beneficial effects of IVIG on relapse rate, rate of new MRI lesions, and disease progression in relapsing-remitting MS. A remyelinating effect of IVIG, as suggested by animal models, was not documented. ${ }^{100-102}$ IVIG is considered to be a second-line therapy for MS. In pediatric MS, treatment with IVIG is used at the dose of $0.4 \mathrm{~g} / \mathrm{kg}$ for 3-5 consecutive days, and administration is repeated monthly or every 3 months. ${ }^{94}$

\section{Immunosuppressive drugs}

Of the following treatments, only mitoxantrone is FDA approved for worsening MS in adults. Its use has not been validated in the pediatric population. The use of immunosuppressive therapies in children with MS has not been reported in the literature, although these drugs are used anecdotally for pediatric patients doing poorly on IFNB or GA. All immunosuppressive drugs have potential serious side effects, specifically in the pediatric population, that include infection, secondary malignancies, and infertility.

Azathioprine. Before IFNB and GA were introduced to the market, azathioprine had been used for years as a mild immunosuppressive drug for adult MS. ${ }^{103-106}$ Azathioprine reduces the rate of relapses in adult MS but evidence is limited for an effect on disease progression. Azathioprine has the potential to induce cytopenia, gastrointestinal intolerance, and liver toxicity. The risk of secondary cancers over the long term has been suggested but never confirmed in adults. The effect of azathioprine on MS MRI activity is unclear. Azathioprine is a second line therapy for patients with relapsing-remitting MS who are intolerant or unwilling to use injectable drugs such as interferon and copolymer. Azathioprine is used in children at the dose of $1-2 \mathrm{mg} / \mathrm{kg} /$ day. No data of 
safety and efficacy of azathioprine are available for pediatric MS.

Cyclophosphamide. Cyclophosphamide is a cytotoxic and immunosuppressive agent, used in systemic autoimmune diseases. Controversial results have been reported on its efficacy in SP MS and RR MS. ${ }^{107-109}$ It seems beneficial when given at early stages of the progressive phase of the disease or in adults doing poorly on IFNB or GA. ${ }^{109-111}$ Cyclophosphamide is typically initiated at the dose of $800 \mathrm{mg} / \mathrm{m}^{2}$ every month for up to 1-2 years. The regimen is adapted to the cell blood count obtained 10-12 days after treatment. Pulses of intravenous glucocorticosteroids can be given concomitantly. Potential adverse effects of cyclophosphamide include infections (particularly if white blood cells are below $1500 / \mathrm{mm}^{3}$ ), nausea, transient alopecia, and gonadal failure. Sperm banking should be considered in males. Hemorrhagic cystitis should be prevented by fluid loading before and after infusions. Finally, there is a risk of bladder and blood cancers, which limits its prescription in the young population. No published report of the use of cyclophosphamide is available for pediatric MS. Lupron is sometimes used for gonad protection in female patients.

Mitoxantrone. A 2-year study with mitoxantrone (Novantrone, Serono) showed decreases in progression of disability, relapse rate, and the number of new MRI lesions in adults with MS compared with placebo. ${ }^{66} \mathrm{Mi}-$ toxantrone is given in adults every 3 months for up to 2 years $\left(12 \mathrm{mg} / \mathrm{m}^{2}\right.$ i.v. each time, maximum cumulative dose of $120 \mathrm{mg} / \mathrm{m}^{2}$ ). Major potential adverse effects of mitoxantrone include cardiotoxicity, leukemia, amenorrhea, and infection. For these reasons, the indication of mitoxantrone is limited to the cases of worsening adult MS resistant to other treatments. There is no published experience of mitoxantrone in the pediatric MS population, although several patients have received this drug for severe MS.

Methotrexate. Oral methotrexate used in association with folic acid is an immunosuppressive agent that may slow the progression of disability in progressive forms of MS in adult patients (7.5-20 mg p.o., once per week). ${ }^{12,113}$ Major side effects include macrocytic anemia and liver and lung toxicity. Methotrexate is rarely used in pediatric MS.

\section{CONCLUSIONS}

Major strides have been made in the past 10 years that have contributed to the improvement of awareness and management of childhood MS. The National Multiple Sclerosis Society has recognized the critical need to provide children and their parents with better care and education and has initiated support for six regional pediatric MS centers across the United States. Further work is needed to better delineate disease course, underlying biological and epidemiological factors that contribute to early MS onset, and differences between ADEM and MS. Finally, as it is unlikely that trials will ever test the efficacy of DMT for MS in children, pediatric neurologists and MS experts will have to combine efforts in the endeavor to improve our understanding of the safety of adult DMT in children.

\section{REFERENCES}

1. Noseworthy JH, Lucchinetti C, Rodriguez M, Weinshenker BG. Multiple sclerosis. N Engl J Med 343:938-952, 2000.

2. Lublin FD, Reingold SC. Defining the clinical course of multiple sclerosis: results of an international survey. Neurology 46:907911, 1996.

3. Medaer R. Does the history of multiple sclerosis go back as far as the 14th century? Acta Neurol Scand 60:189-192, 1979.

4. Shaw CM, Alvord EC. Multiple sclerosis beginning in infancy. $J$ Child Neurol 2:252-256, 1987.

5. Sindern E, Haas J, Stark E, Wurster U. Early onset MS under the age of 16: clinical and paraclinical features. Acta Neurol Scand 86:280-284, 1992.

6. Mikaeloff Y, Suissa S, Vallee L, Lubetzki C, Ponsot G, Confavreux $\mathrm{C}$ et al. First episode of acute CNS inflammatory demyelination in childhood: prognostic factors for multiple sclerosis and disability. $J$ Pediatr 144:246-252, 2004.

7. Poser CM, Paty DW, Scheinberg L, McDonald WI, Davis FA, Ebers GC et al. New diagnostic criteria for multiple sclerosis: guidelines for research protocols. Ann Neurol 13:227-231, 1983.

8. McDonald WI, Compston A, Edan G, Goodkin D, Hartung HP, Lublin $\mathrm{F}$ et al. Recommended diagnostic criteria for multiple sclerosis: guidelines from the International Panel on the diagnosis of multiple sclerosis. Ann Neurol 50:121-127, 2001.

9. Kermode AG, Thompson AJ, Tofts P, MacManus DG, Kendall BE, Kingsley DP et al. Breakdown of the blood-brain barrier precedes symptoms and other MRI signs of new lesions in multiple sclerosis. Brain 113:1477-1489, 1990.

10. Hahn CD, Shroff MM, Blaser SI, Banwell BL. MRI criteria for multiple sclerosis: evaluation in a pediatric cohort. Neurology 62:806-808, 2004.

11. Hauser SL, Bresnan MJ, Reinherz MJ, Weiner HL. Childhood multiple sclerosis: clinical features and demonstration of changes in T cell subsets with disease activity. Ann Neurol 11s:463-468, 1982.

12. Duquette P., Murray TJ, Pleines J, Ebers GC, Sadovnick D, Weldon P et al. Multiple sclerosis in childhood: clinical profile in 125 patients. J Pediatr 111:359-363, 1987.

13. Bauer HJ, Hanefeld F, Christen HJ. Multiple sclerosis in early childhood. Lancet 336:1190, 1990.

14. Hanefeld F, Bauer HJ, Christen HJ, Kruse B, Bruhn H, Frahm J Multiple sclerosis in childhood: report of 15 cases. Brain Dev 13:410-416, 1991

15. Perniola T, Russo MG, Margari L, Buttiglione M, Simone IL. Multiple sclerosis in childhood: longitudinal study in 14 cases. Acta Neurol (Napoli) 13:236-248, 1991.

16. Ghezzi A, Deplano V, Faroni J, Grasso MG, Liguori M, Marrosu $\mathrm{G}$ et al. Multiple sclerosis in childhood: clinical features of 149 cases. Mult Scler 3:43-46, 1997.

17. Pinhas-Hamiel O, Barak Y, Siev-Ner I, Achiron A. Juvenile multiple sclerosis: clinical features and prognostic characteristics. J Pediatr 132:735-737, 1998.

18. Ruggieri M., Polizzi A, Pavone L, Grimaldi LM. Multiple sclerosis in children under 6 years of age. Neurology 53:478-484, 1999.

19. Dale RC., de Sousa C, Chong WK, Cox TC, Harding B, Neville BG. Acute disseminated encephalomyelitis, multiphasic disseminated encephalomyelitis and multiple sclerosis in children. Brain 123:2407-2422, 2000.

20. Ruggieri M, Iannetti P, Polizzi A, Pavone L, Grimaldi LM, Italian Society of Paediatric Neurology Study Group on Childhood Mul- 
tiple Sclerosis: multiple sclerosis in children under 10 years of age. Neurol Sci 25 (Suppl 4):S326-S335, 2004.

21. Ghezzi A. Childhood-juvenile multiple sclerosis: clinical characteristics and treatment. Expert Rev Neurother 5:403-411, 2005.

22. Rose AS, Ellison GW, Myers LW, Tourtelotte WW. Criteria for the clinical diagnosis of multiple sclerosis. Neurology 26:20-22, 1976.

23. Trojano M, Paolicelli D, Bellacosa A, Fuiani A, Cataldi S, Di Monte E. Atypical forms of multiple sclerosis or different phases of a same disease? Neurol Sci 25 (Suppl 4):S323-S325, 2004.

24. Ghezzi A. Clinical characteristics of multiple sclerosis with early onset. Neurol Sci 25:S336-S339, 2004.

25. Lucchinetti C, Bruck W, Parisi J, Scheithauer B, Rodriguez M, Lassmann $\mathrm{H}$. Heterogeneity of multiple sclerosis lesions: implications for the pathogenesis of demyelination. Ann Neurol 47: 707-717, 2000.

26. Banwell B, Kennedy J, Krupp L, Bar-Or A, Tenembaum S, Belman A et al. Clinical and demographic features of pediatric MS: preliminary data from a multinational collaborative study. Neurology (Suppl 5) 62:A230, 2004.

27. Cole GF., Auchterlonie LA, Best PV. Very early onset multiple sclerosis. Dev Med Child Neurol 37:667-672, 2005.

28. Banwell BL. Anderson PE. The cognitive burden of multiple sclerosis in children. Neurology 64:891-894, 2005.

29. MacAllister WS, Belman AL, Milazzo M, Weisbrot DM, Christodoulou C, Scherl WF et al. Cognitive functioning in children and adolescents with multiple sclerosis. Neurology 64:1422$1425,2005$.

30. Hahn CD., Miles BS, Macgregor DL, Blaser SI, Banwell BL, Hetherington CR. Neurocognitive outcome after acute disseminated encephalomyelitis. Pediatr Neurol 29:117-123, 2003.

31. Baoxun ZDL, Weiming H, Xiugun L, Shihe L, Yinchang Y, Sinmei J et al. MS in China: a clinical study of 256 cases. In: Multiple Sclerosis East and West (Kuroiwa LKY, ed), pp 71-81. Fukuoka, Japan: Kyushu University Press, 1982.

32. Higa H, Toyonaga K, Nishihara T, Sakugawa H, Ikuta F. MS in Okinawa. In: Multiple Sclerosis East and West (Kuroiwa LKY, ed), pp 49-56. Fukuoka, Japan: Kyushu University Press, 1982.

33. Hung TP. MS in Taiwan: a reappraisal. In: Multiple Sclerosis East and West (Kuroiwa LKY, ed), pp 83-96. Fukuoka, Japan: Kyushu University Press, 1982.

34. Kim SW, Kim SK. Multiple sclerosis in Busan Korea: clinical features and prevalence. In: Multiple Sclerosis East and West (Kuroiwa LKY, ed), pp 57-80. Fukuoka, Japan: Kyushu University Press, 1982.

35. Shiraishi K, Higuchi Yozawa K, Hao Q, Saida T. Clinical course and prognosis of 27 patients with childhood onset multiple sclerosis in Japan. Brain Dev 27:224-227, 2005.

36. Elian M, Nightingale S, Dean G. Multiple sclerosis among United Kingdom-born children of immigrants from the Indian subcontinent, Africa and the West Indies. J Neurol Neurosurg Psychiatry 53:906-911, 1990.

37. Zelnik N., Gale AD, Shelburne SA Jr. Multiple sclerosis in black children. J Child Neurol 6:53-57, 1991.

38. Mikaeloff Y, Adamsbaum C, Husson B, Vallee L, Ponsot G, Confavreux $\mathrm{C}$, et al.: MRI prognostic factors for relapse after acute CNS inflammatory demyelination in childhood. Brain 127: 1942-1947, 2004.

39. Triulzi F, Scotti G. Differential diagnosis of multiple sclerosis: contribution of magnetic resonance techniques. J Neurol Neurosurg Psychiatry 64 (Suppl 1):S6-S14, 1998.

40. Hynson JL., Kornberg AJ, Coleman LT, Shield L, Harvey AS, Kean MJ. Clinical and neuroradiologic features of acute disseminated encephalomyelitis in children. Neurology 56:1308-1312, 2001.

41. Pohl D, Rostasy K, Reiber H, Hanefeld F. CSF characteristics in early-onset multiple sclerosis. Neurology 63:1966-1967, 2004.

42. Alotaibi S, Kennedy J, Tellier R, Stephens D, Banwell B. Epstein-Barr virus in pediatric multiple sclerosis. JAMA 291:18751879, 2004.

43. Andersson PB, Goodkin DE. Glucocorticosteroid therapy for multiple sclerosis: a critical review. J Neurol Sci 160:16-25, 1998.
44. Brusaferri F, Candelise L. Steroids for multiple sclerosis and optic neuritis: a meta-analysis of randomized controlled clinical trials. J Neurol 247:435-442, 2000.

45. Goodin DS, Frohman EM, Garmany GP Jr, Halper J, Likosky WH, Lublin FD, et al. Disease modifying therapies in multiple sclerosis: report of the Therapeutics and Technology Assessment Subcommittee of the American Academy of Neurology and the MS Council for Clinical Practice Guidelines. Neurology 58:169_ 178, 2002.

46. Beck RW. The optic neuritis treatment trial: three-year follow-up results. Arch Ophthalmol 113:136-137, 1995.

47. Oliveri RL, Valentino P, Russo C, Sibilia G, Aguglia U, Bono F, et al. Randomized trial comparing two different high doses of methylprednisolone in MS: a clinical and MRI study. Neurology 50:1833-1836, 1998.

48. Miller DM, Weinstock-Guttman B, Bethoux F, Lee JC, Beck G, Block V, et al. A meta-analysis of methylprednisolone in recovery from multiple sclerosis exacerbations. Mult Scler 6:267-273, 2000.

49. Weinshenker BG, O’Brien PC, Petterson TM, Noseworthy JH, Lucchinetti CF, Dodick DW et al. A randomized trial of plasma exchange in acute central nervous system inflammatory demyelinating disease. Ann Neurol 46:878-886, 1999.

50. Takahashi I, Sawaishi Y, Takeda O, Enoki M, Takada G. Childhood multiple sclerosis treated with plasmapheresis. Pediatr Neurol 17:83-87, 1997.

51. Finsterer J, Grass R, Stollberger C, Mamoli B. Immunoglobulins in acute, parainfectious, disseminated encephalo-myelitis. Clin Neuropharmacol 21:258-261, 1998.

52. Nishikawa M, Ichiyama T, Hayashi T, Ouchi K, Furukawa S. Intravenous immunoglobulin therapy in acute disseminated encephalomyelitis. Pediatr Neurol 21:583-586, 1999.

53. Apak RA, Anlar B, Saatci I. A case of relapsing acute disseminated encephalomyelitis with high dose corticosteroid treatment. Brain Dev 21:279-282, 1999.

54. Pradhan S, Gupta RP, Shashank S, Pandey N. Intravenous immunoglobulin therapy in acute disseminated encephalomyelitis. J Neurol Sci 165:56-61, 1999.

55. Sahlas DJ, Miller SP, Guerin M, Veilleux M, Francis G. Treatment of acute disseminated encephalomyelitis with intravenous immunoglobulin. Neurology 54:1370-1372, 2000.

56. Noseworthy JH, O'Brien PC, Petterson TM, Weis J, Stevens L, Peterson WK et al. A randomized trial of intravenous immunoglobulin in inflammatory demyelinating optic neuritis. Neurology 56:1514-1522, 2001

57. Sorensen PS, Haas J, Sellebjerg F, Olsson T, Ravnborg M. IV immunoglobulins as add-on treatment to methylprednisolone for acute relapses in MS. Neurology 63:2028-2033, 2004.

58. The IFNB Multiple Sclerosis Study Group. Interferon beta- $1 \mathrm{~b}$ is effective in relapsing-remitting multiple sclerosis. I. Clinical results of a multicenter, randomized, double-blind, placebo-controlled trial. Neurology 43:655-661, 1993.

59. Johnson KP, Brooks BR, Cohen JA, Ford CC, Goldstein J, Lisak $\mathrm{RP}$ et al. Copolymer 1 reduces relapse rate and improves disability in relapsing-remitting multiple sclerosis: results of a phase III multicenter, double-blind placebo-controlled trial. The Copolymer 1 Multiple Sclerosis Study Group. Neurology 45:1268-1276, 1995.

60. Jacobs LD, Cookfair DL, Rudick RA, Herndon RM, Richert JR, Salazar AM et al. Intramuscular interferon beta-1a for disease progression in relapsing multiple sclerosis. The Multiple Sclerosis Collaborative Research Group (MSCRG). Ann Neurol 39: 285-294, 1996.

61. PRISMS (Prevention of Relapses and Disability by Interferon beta-1a Subcutaneously in Multiple Sclerosis) Study Group. Randomised double-blind placebo-controlled study of interferon beta-1a in relapsing/remitting multiple sclerosis. Lancet 352:1498 1504,1998

62. Comi G, Filippi M, Wolinsky JS. European/Canadian multicenter, double-blind, randomized, placebo-controlled study of the effects of glatiramer acetate on magnetic resonance imagingmeasured disease activity and burden in patients with relapsing 
multiple sclerosis. European/Canadian Glatiramer Acetate Study Group. Ann Neurol 49:290-297, 2001.

63. Jacobs LD, Beck RW, Simon JH, Kinkel RP, Brownscheidle CM, Murray TJ et al. Intramuscular interferon beta-1a therapy initiated during a first demyelinating event in multiple sclerosis. CHAMPS Study Group. N Engl J Med 343:898-904, 2000.

64. Comi G, Filippi M, Barkhof F, Durelli L, Edan G, Fernandez O et al. Effect of early interferon treatment on conversion to definite multiple sclerosis: a randomised study. Lancet 357:1576-1582, 2001.

65. PRISMS Study Group and the University of British Columbia MS/MRI Analysis Group. PRISMS-4: Long-term efficacy of interferon-beta-1a in relapsing MS. Neurology 56:1628-1636, 2001.

66. Hartung HP, Gonsette R, Konig N, Kwiecinski H, Guseo A, Morrissey SP et al. Mitoxantrone in progressive multiple sclerosis: a placebo-controlled, double-blind, randomised, multicentre trial. Lancet 360:2018-2025, 2002.

67. Van Assche G, Van Ranst M, Sciot R, Dubois B, Vermeire S, Noman et al. Progressive multifocal leukoencephalopathy after natalizumab therapy for Crohn's disease. N Engl J Med 28:362$368,2005$.

68. Kleinschmidt-DeMasters BK, Tyler KL. Progressive multifocal leukoencephalopathy complicating treatment with natalizumab and interferon beta-1a for multiple sclerosis. $N$ Engl J Med 28: 369-374, 2005.

69. Langer-Gould A, Atlas SW, Green AJ, Bollen AW, Pelletier D. Progressive multifocal leukoencephalopathy in a patient treated with natalizumab. $N$ Engl J Med 353:375-381, 2005.

70. Miller DH, Khan OA, Sheremata WA, Blimhardt LD, Rice GP, Libonati MA et al. A controlled trial of natalizumab for relapsing multiple sclerosis. $N$ Engl J Med 348:15-23, 2003.

71. Polman C, O'Connor P, Havrdova E, et al. Clinical results from AFFIRM: a randomized, double-blind, placebo-controlled, multicenter trial to determine the efficacy and safety of natalizumab in patients with relapsing remitting multiples sclerosis (MS). Neurology 64 (Suppl 1):A146, 2005.

72. O'Connor P, Havrdova E, Hutchinson M, et al. Safety, tolerability and immunogenicity of natalizumab: results from the AFFIRM trial. Neurology 64 (Suppl 1):A146, 2005.

73. Miller D, O'Connor P, Havrdova E, et al. The efficacy of natalizumab on magnetic resonance imaging measures in patients with relapsing remitting multiple sclerosis: results from the AFFIRM trial. Neurology 64 (Suppl 1):A146, 2005.

74. Rudick R, Stuart W, Calabresi P, et al. SENTINEL: a randomized, double-blind, placebo-controlled, multicenter trial to determine the efficacy and safety of natalizumab, when added to intramuscular interferon beta-1a, in patients with relapsing multiple sclerosis. One year clinical and MRI results. Neurology 64 (Suppl 1):A276, 2005.

75. Calabresi P, Stuart W, Confavreux C, et al. Safety and tolerability of natalizumab: results from the SENTINEL study. Neurology 64 (Suppl 1):A277, 2005.

76. Kappos L, Polman C, Pozzilli C, Thompson A, Beckmann K, Dahlke F. Final analysis of the European multicenter trial on IFNbeta-1b in secondary-progressive MS. Neurology 57:19691975, 2001.

77. Li DK, Zhao GJ, Paty DW. Randomized controlled trial of interferon-beta-1a in secondary progressive MS: MRI results. Neurology $56: 1505-1513,2001$.

78. Secondary Progressive Efficacy Clinical Trial of Recombinant Interferon-beta-1a in MS (SPECTRIMS) Study Group. Randomized controlled trial of interferon-beta-1a in secondary progressive MS: clinical results. Neurology 56:1496-1504, 2001.

79. Cohen JA, Cutter GR, Fischer JS, Goodman AD, Heidenreich FR, Kooijmans MF et al. Benefit of interferon beta-1a on MSFC progression in secondary progressive MS. Neurology 59:679687, 2002.

80. Leary SM, Miller DH, Stevenson VL, Brex PA, Chard DT, Thompson AJ. Interferon beta-1a in primary progressive MS: an exploratory, randomized, controlled trial. Neurology 60:44-51, 2003.

81. Kita M, Cohen J, Fox B et al. A phase II trial of mitoxantrone in patients with primary progressive MS: an exploratory, randomized, controlled trial. Neurology 62 (Suppl 5):A99, 2004.

82. Wolinsky J, Pardo L, Stark Y et al. Effect of glatiramer acetate on primary progressive multiple sclerosis: initial analysis of the completed PROMiSe trial. Neurology 62 (Suppl 5):A97, 2004.

83. Hommes OR, Sorensen PS, Fazekas F, Enriquez MM, Koelmel $\mathrm{HW}$, Fernandez $\mathrm{O}$ et al. Intravenous immunoglobulin in secondary progressive multiple sclerosis: randomised placebo-controlled trial. Lancet 364:1149-1156, 2004.

84. Tenmbaum S, Banwell B, Boiko A et al. Treatment of childhood and adolescent multiple sclerosis with interferon beta-1b. Neurology 64 (Suppl 1):A384, 2005.

85. Adams AB, Tyor WR, Holden KR. Interferon beta- $1 \mathrm{~b}$ and childhood multiple sclerosis. Pediatr Neurol 21:481-483, 1999.

86. Schilling S, Haertel C, Sperner J Follow-up of interferon beta-1b treatment in patients with secondary progressive multiple sclerosis. Neuropediatrics 33:A31, 2002.

87. Waubant E, Hietpas J, Stewart T, Dyme Z, Herbert J, Lacy J et al. Interferon beta-1a in children with multiple sclerosis is well tolerated. Neuropediatrics 32:211-213, 2001.

88. Mikaeloff Y, Moreau T, Debouverie M, Pelletier J, Lebrun C, Gout $\mathrm{O}$ et al. Interferon-beta treatment in patients with childhoodonset multiple sclerosis. J Pediatr 139:443-446, 2001.

89. Ghezzi A, Ruggieri M, Trojano M, Filippi M. Italian studies on early-onset multiple sclerosis: the present and the future. Neurol Sci 25 (Suppl 4):S346-S349, 2004.

90. Pohl D, Rostasy K, Gartner J, Hanefeld F. Treatment of early onset multiple sclerosis with subcutaneous interferon beta-1a. Neurology 64:888-890, 2005.

91. Tenembaum S, Segura M. Interferon beta-1a is effective and well tolerated in paediatric patients: results of a 6-year study. Multiple Scler (in press).

92. Etheridge LJ, Beverley DW, Ferrie C, McManus E. The use of interferon beta in relapsing-remitting multiple sclerosis. Arch Dis Child 89:789-791, 2004.

93. Kornek B, Bernert G, Balassy C, Geldner J, Prayer D, Feucht M. Glatiramer acetate treatment in patients with childhood and juvenile onset multiple sclerosis. Neuropediatrics 34:120-126, 2003.

94. Krupp LB, Macallister WS. Treatment of pediatric multiple slerosis. Curr Treat Options Neurol 7:191-199, 2005.

95. Yong VW. Differential mechanisms of action of interferon-beta and glatiramer acetate in MS. Neurology 59:802-808, 2002.

96. Durelli L, Verdun E, Barbero P, Bergui M, Versino E, Ghezzi A et al. Independent Comparison of Interferon (INCOMIN) Trial Study Group: every-other-day interferon beta- $1 \mathrm{~b}$ versus onceweekly interferon beta-1a for multiple sclerosis: results of a 2 -year prospective randomised multicentre study (INCOMIN). Lancet 359:1453-1460, 2002.

97. Panitch H, Goodin DS, Francis G, Chang P, Coyle PK, O'Connor $P$ et al. EVIDENCE Study Group. EVidence of Interferon Doseresponse: Europian North American Compartative Efficacy; University of British Columbia MS/MRI Research Group. Randomized, comparative study of interferon beta-1a treatment regimens in MS: The EVIDENCE Trial. Neurology 59:1496-1506, 2002.

98. PRISMS Study Group. Francis GS, Rice GP, Alsop JC, Interferon beta-1a in MS: results following development of neutralizing antibodies in PRISMS. Neurology 65:48-55, 2005.

99. Banwell B. Treatment of children and adolescents with multiple sclerosis. Expert Rev Neurother 5:391-401, 2005.

100. Fazekas F, Deisenhammer F, Strasser-Fuchs S, Nahler G, Mamoli B. Randomised placebo-controlled trial of monthly intravenous immunoglobulin therapy in relapsing-remitting multiple sclerosis. Lancet 349:589-593, 1997.

101. Achiron A, Gabbay U, Gilad R., Hassin-Baer S, Barak Y, Gornish $\mathrm{M}$ et al. Intravenous immunoglobulin treatment in multiple sclerosis: effect on relapses. Neurology 50:398-402, 1998.

102. Sorensen PS, Wanscher B, Jensen CV, Schreiber K, Blinkenberg $\mathrm{M}$, Ravnborg $\mathrm{M}$ et al. Intravenous immunoglobulin $\mathrm{G}$ reduces MRI activity in relapsing multiple sclerosis. Neurology 50:12731281, 1998.

103. Goodkin DE, Bailly RC, Teetzen ML, Hertsgaard D, Beatty WW. 
The efficacy of azathioprine in relapsing-remitting multiple sclerosis. Neurology 41:20-25, 1991.

104. Yudkin PL, Ellison GW, Ghezzi A, Goodkin DE, Hughes RA, McPherson $\mathrm{K}$ et al. Overview of azathioprine treatment in multiple sclerosis. Lancet 338:1051-1055, 1991.

105. Palace J, Rothwell P. New treatments and azathioprine in multiple sclerosis. Lancet 350:261, 1997.

106. Cavazzuti M, Merelli E, Tassone G, Mavilla L. Lesion load quantification in serial MR of early relapsing multiple sclerosis patients in azathioprine treatment: a retrospective study. Eur Neurol 38:284-290, 1997.

107. Hauser SL, Dawson DM, Lehrich JR, Beal MF, Kevy SV, Propper $\mathrm{RD}$ et al. Intensive immunosuppression in progressive multiple sclerosis: a randomized, three-arm study of high-dose intravenous cyclophosphamide, plasma exchange, and ACTH. $N$ Engl J Med 308:173-180, 1983.

108. The Canadian Cooperative Multiple Sclerosis Study Group. The Canadian cooperative trial of cyclophosphamide and plasma exchange in progressive multiple sclerosis. Lancet 337:441-446, 1991.

109. Weiner HL, Mackin GA, Orav EJ, Hafler DA, Dawson DM, LaPierre $\mathrm{Y}$ et al. Intermittent cyclophosphamide pulse therapy in progressive multiple sclerosis: final report of the Northeast Cooperative Multiple Sclerosis Treatment Group. Neurology 43:910-918, 1993.

110. Gobbini MI, Smith ME, Richert ND, Frank JA, McFarland HF. Effect of open label pulse cyclophosphamide therapy on MRI measures of disease activity in five patients with refractory relapsing-remitting multiple sclerosis. J Neuroimmunol 99:142149, 1999.

111. Smith DR, Weinstock-Guttman B, Cohen JA, Wei X, Gutmann $\mathrm{C}$, Bakshi R, Olek M et al. A randomized blinded trial of combination therapy with cyclophosphamide in patients-with active multiple sclerosis on interferon beta. Mult Scler 11:573-582, 2005.

112. Goodkin DE, Rudick RA, VanderBrug Medendorp S, Daughtry MM, Schwetz KM et al. Low-dose $(7.5 \mathrm{mg}$ ) oral methotrexate reduces the rate of progression in chronic progressive multiple sclerosis. Ann Neurol 37: 30-40, 1995.

113. Goodkin DE, Rudick RA, VanderBrug Medendorp S, Daughtry MM, Van Dyke C. Low-dose oral methotrexate in chronic progressive multiple sclerosis: analyses of serial MRIs. Neurology 47: 1153-1157, 1996. 